\title{
CHARACTERIZATIONS OF $\mathbb{P}^{N}$ IN ARBITRARY CHARACTERISTIC*
}

\section{YASUYUKI KACHI ${ }^{\dagger}$ AND JÁNOS KOLLÁR ${ }^{\ddagger}$}

There are several results which characterize $\mathbb{P}^{n}$ among projective varieties. The first substantial theorem in this direction is due to [H-K57], using the topological properties of $\mathbb{C P}^{n}$. Later characterizations approached $\mathbb{P}^{n}$ as the projective variety with the most negative canonical bundle. The first such theorem is due to [K-O72] and later it was generalized in the papers [Ionescu86, Fujita87]. A characterization using the tangent bundle was given in [Mori79, Siu-Yau80]. Other characterizations using vector bundles are given in the papers [Ye-Zhang90, Sato99, Kachi99].

The results of [K-O72, Ionescu86, Fujita87] ultimately relied on the Kodaira vanishing theorem, thus they were restricted to characteristic zero. The aim of this note is to provide an argument which does not use higher cohomologies of line bundles. This makes the proofs work in arbitrary characteristic. Thus (1) generalizes [Ionescu86, Fujita87] and (2) is a characteristic free version of [K-O72]. The proofs of [Ionescu86, Fujita87] work if we know enough about extremal contractions of smooth varieties. Thus the results have been known in any characteristic for surfaces and they can be read off from the classification of contractions of smooth 3-folds in positive characteristic developed in the papers [Kollár91, S-B97, Megyesi98].

For the rest of the paper all varieties are over an algebraically closed field of arbitrary characteristic.

THEOREm 1. Let $X$ be a smooth projective variety of dimension $n$ and $H$ an ample divisor on $X$. Then the pair $(X, H)$ satisfies one of the following:

1. $(n-1) H+K_{X}$ is nef.

2. $X \cong \mathbb{P}^{n}$ and $H$ is a hyperplane section.

3. $X$ is a quadric in $\mathbb{P}^{n+1}$ and $H$ is a hyperplane section.

4. $X$ is the projectivization of a rank $n$ vector bundle over a smooth curve $A$ and $H=\mathcal{O}(1)$.

With a little bit of work on the scrolls in (1.4) this implies the following characterization of projective spaces and hyperquadrics.

CoROllary 2. Let $X$ be a smooth projective variety of dimension $n$.

1. $X$ is isomorphic to $\mathbb{P}^{n}$ iff there is an ample divisor $H$ such that $-K_{X}$ is numerically equivalent to $(n+1) H$.

2. $X$ is isomorphic to a hyperquadric $\mathbb{Q}^{n} \subset \mathbb{P}^{n+1}$ iff there is an ample divisor $H$ such that $-K_{X}$ is numerically equivalent to $n H$.

Our proof of (1) also yields the following:

Corollary 3. Let $X$ be a smooth projective variety of dimension n. Assume that $K_{X}$ has negative intersection number with some curve. Then $X$ is isomorphic to $\mathbb{P}^{n}$ iff

1. $\left(-K_{X} \cdot C\right) \geq n+1$ for every rational curve $C \subset X$, and

2. $\left(-K_{X}\right)^{n} \geq(n+1)^{n}$.

\footnotetext{
${ }^{*}$ Received March 29, 2000; accepted for publication June 2, 2000.

†Department of Mathematics, Johns Hopkins University, Baltimore MD 21218, USA (kachi@ math.jhu.edu).

$\ddagger$ Department of Mathematics, Princeton University; Princeton NJ 08544-1000, USA (kollar@math.princeton.edu).
} 
It is conjectured that (3.1) alone characterizes $\mathbb{P}^{n}$. It has also been conjectured that (3.2) also characterizes $\mathbb{P}^{n}$ among Fano varieties. This, however, turned out to be false for $n \geq 4$ [Batyrev82].

The first step of the proof of (1) is to identify the family of lines on $X$. The results of [Mori79] show that there is a family of rational curves $\left\{C_{t}\right\}$ on $X$ such that $\left(H \cdot C_{t}\right)=1$ and this family has the expected dimension. Focusing on these rational curves leads to other characterizations of $\mathbb{P}^{n}$ and of $\mathbb{Q}^{n}$, which strengthen (2) in characteristic zero.

THEOREM 4. Let $X$ be a smooth projective variety of dimension $n$ over $\mathbb{C}$ and $L$ an ample line bundle on $X$.

1. [A-B-W93] Assume that for every $x, y \in X$ there is a rational curve $C_{x y}$ through $x, y$ with $\left(L \cdot C_{x y}\right)=1$. Then $X \cong \mathbb{P}^{n}$ and $L \cong \mathcal{O}(1)$.

2. [Kachi-Sato99] Assume that for every $x, y, z \in X$ there is a rational curve $C_{x y z}$ through $x, y, z$ with $\left(L \cdot C_{x y z}\right)=2$. Then $X \cong \mathbb{P}^{n}$ or $X \cong \mathbb{Q}^{n}$ and $L \cong \mathcal{O}(1)$.

The positive characteristic version of (4) is still open. Concentrating on higher degree curves leads to the following characterization of Veronese varieties, which gives another generalization of (2.1) and also leads to a weaker version of (1).

ThEOREM 5. [Kachi99] Let $X$ be a smooth projective variety of dimension $n$ (over an algebraically closed field of arbitrary characteristic) and $L$ an ample divisor on $X$. Assume that:

1. For general $x, y \in X$ there is a connected (possibly reducible) curve $C_{x y}$ through $x, y$ with $\left(L \cdot C_{x y}\right) \leq d$.

2. For every irreducible subvariety $Z \subset X$ of dimension at least 2 we have $\left(L^{\operatorname{dim} Z} \cdot Z\right) \geq d^{\operatorname{dim} Z}$.

3. Fujita's sectional genus of $\frac{1}{d} L$ is less than 1 , that is,

$$
\left(L^{n-1} \cdot\left(\frac{n-1}{d} L+K_{X}\right)\right)<0 .
$$

Then $X \cong \mathbb{P}^{n}$ and $L \cong \mathcal{O}(d)$.

6 (Proof of (1)). As we noted, we may assume that $n \geq 3$. We are done if $K_{X}+(n-1) H$ is nef.

If $K_{X}+(n-1) H$ is not nef, then by the cone theorem of Mori (cf. [Kollár95, III.1]) there is a $c>0$ and a rational curve $C \subset X$ such that

1. $K_{X}+(n-1+c) H$ is nef,

2. $C \cdot\left(K_{X}+(n-1+c) H\right)=0$, and

3. $-\left(C \cdot K_{X}\right) \leq n+1$.

Thus we see that

$$
(C \cdot H) \leq \frac{-\left(C \cdot K_{X}\right)}{n-1+c} \leq \frac{n+1}{n-1+c}<2 .
$$

Hence $C \cdot H=1$ and $-\left(C \cdot K_{X}\right)=n$ or $n+1$. Let $f: \mathbb{P}^{1} \rightarrow X$ be the normalization of $C, 0 \in \mathbb{P}^{1}$ a point and $x=f(0)$. Then by [Kollár95, II.1]

$$
\begin{array}{ll}
\operatorname{dim}_{[f]} \operatorname{Hom}\left(\mathbb{P}^{1}, X\right) & \geq-\left(C \cdot K_{X}\right)+n, \quad \text { and } \\
\operatorname{dim}_{[f]} \operatorname{Hom}\left(\mathbb{P}^{1}, X, 0 \mapsto x\right) & \geq-\left(C \cdot K_{X}\right) .
\end{array}
$$

We consider 3 separate cases corresponding to the three outcomes (1.2-4). 
7 (Case 1: $\left.-\left(C \cdot K_{X}\right)=n+1\right)$. In order to go from homomorphisms to curves in $X$, we have to quotient out by the automorphism group. Thus $\operatorname{Hom}\left(\mathbb{P}^{1}, X, 0 \mapsto x\right)$ gives an $(n-1)$-dimensional family of rational curves through $x \in X$ since $\operatorname{Aut}\left(\mathbb{P}^{1}, 0\right)$ is 2-dimensional. This implies that the Picard number of $X$ is 1 (cf. [Kollár95, IV.3.13.3]). Thus $-K_{X}$ is numerically equivalent to $(n+1) H$. The following lemma shows that $X \cong \mathbb{P}^{n}$. We formulate it for singular varieties, the setting needed for the third case.

LEMMA 8. Let $X$ be a normal, projective variety and $x \in X$ a smooth point. Let $H$ be an ample Cartier divisor on $X$ and let $\left\{C_{t}: t \in T\right\}$ be an $(n-1)$-dimensional family of curves through $x$. Assume that $\left(C_{t} \cdot H\right)=1$ and $\left(-K_{X} \cdot H^{n-1}\right)>(n-1)\left(H^{n}\right)$. Then $X \cong \mathbb{P}^{n}, H=\mathcal{O}(1)$ and the $C_{t}$ are lines through $x$.

Proof. By Riemann-Roch,

$$
h^{0}\left(X, \mathcal{O}_{X}(m H)\right)=m^{n} \frac{\left(H^{n}\right)}{n !}+m^{n-1} \frac{-\left(K_{X} \cdot H^{n-1}\right)}{2(n-1) !}+O\left(m^{n-2}\right) .
$$

For a section of a line bundle it is

$$
\left(\begin{array}{c}
m+n-1 \\
n
\end{array}\right)=m^{n} \frac{1}{n !}+m^{n-1} \frac{n-1}{2(n-1) !}+O\left(m^{n-2}\right)
$$

conditions to vanish at a smooth point $x \in X$ to order $m$. Comparing the two estimates we see that for large $m$

$$
\begin{array}{lll}
H^{0}\left(X, \mathcal{O}_{X}(m H) \otimes I_{x}^{m+1}\right) & \geq \text { const }) \cdot m^{n} & \text { if }\left(H^{n}\right)>1, \text { and } \\
H^{0}\left(X, \mathcal{O}_{X}(m H) \otimes I_{x}^{m}\right) & \geq \text { (const) } \cdot m^{n-1} & \text { if }\left(H^{n}\right)=1 .
\end{array}
$$

Pick any member $D \in\left|\mathcal{O}_{X}(m H) \otimes I_{x}^{m+1}\right|$ (resp. $D \in\left|\mathcal{O}_{X}(m H) \otimes I_{x}^{m}\right|$ ) and take $C_{t} \not \subset D$. Then $\left(C_{t} \cdot D\right)=m$. On the other hand, $\left(C_{t} \cdot D\right) \geq \sum_{y \in C_{t}} \operatorname{mult}_{y} D \geq m+1$ (resp. $\geq m$ ). This is a contradiction if $\left(H^{n}\right)>1$. If $\left(H^{n}\right)=1$ then $\left(C_{t} \cap D\right)=\{x\}$ and the linear system $\left|\mathcal{O}_{X}(m H) \otimes I_{x}^{m}\right|$ is constant along $C_{t}$.

By varying $x \in C_{t}$, we also see that the line bundle $\mathcal{O}_{C_{t}}(m H)$ has a section with an $m$-fold zero at a general point of $C_{t}$. Since $\operatorname{deg} \mathcal{O}_{C_{t}}(m H)=m$, this implies that a general $C_{t}$ is a smooth rational curve.

Set $Y=B_{x} X$ with exceptional divisor $E$ and projection $\pi: Y \rightarrow X$. Set $M=\pi^{*} H-E$. Then $H^{0}\left(\mathcal{O}_{Y}(m M)\right) \cong H^{0}\left(X, \mathcal{O}_{X}(m H) \otimes I_{x}^{m}\right)$, hence $h^{0}\left(\mathcal{O}_{Y}(m M)\right) \geq$ (const) $\cdot m^{n-1}$. For large $m$, let $p: Y \rightarrow Z$ denote the map induced by $|m M|$. Up to birational equaivalence, $p$ does not depend on $m$ and it has connected 1-dimensional general fibers. Moreover, for any subset $V \subset Y$ of dimension at most $n-2$ there is a member $D \in|m M|$ containing $V$.

Every curve $C_{t}$ lifts to a curve $C_{t}^{\prime}$ on $Y$ and there is an open subset $T^{0} \subset T$ such that the curves $\left\{C_{t}^{\prime}: t \in T^{0}\right\}$ form a single algebraic family. (In our case these correspond to the curves $C_{t}$ which are smooth at $x$.) By shrinking $T$ we pretend that $T=T^{0}$. Note that $\left(C_{t}^{\prime} \cdot E\right)=1$ and $\left(C_{t}^{\prime} \cdot M\right)=0$.

We may assume that $Z$ is proper. Let $V \subset Y$ be the set where $p$ is not defined and choose $V \subset D \in|m M|$.

If a curve $C_{t}^{\prime}$ passes through $V$ then $C_{t}^{\prime} \subset D$ since $\left(M \cdot C_{t}^{\prime}\right)=0$. The curves $C_{t}^{\prime}$ cover an open subset of $Y$, hence a general $C_{t}^{\prime}$ is disjoint from $D$. Thus $p$ is defined everywhere along a general $C_{t}^{\prime}$ and $C_{t}^{\prime}$ is a fiber of $p$ (at least set theoretically). Hence there are open subsets $Y^{0} \subset Y$ and $Z^{0} \subset Z$ such that $p^{0}: Y^{0} \rightarrow Z^{0}$ is proper and 
flat. $\left(E \cdot C_{t}^{\prime}\right)=1$, thus $E$ is a rational section of $p$. In particular, a general fiber of $p$ is reduced. Thus, possibly after shrinking $Y^{0}$, we may assume that $p^{0}$ is a $\mathbb{P}^{1}$-bundle. Set $E^{0}:=E \cap Y^{0}$.

$Z^{0}$ parametrizes curves in $Y$ and by looking at the image of these curves in $X$ we obtain a morphism $h: Z^{0} \rightarrow \operatorname{Chow}(X)$. (See [Kollár95, I.3-4] for the definition of Chow and its basic properties.) Let $Z^{\prime}$ be the normalization of the closure of the image of $Z^{0}$ in Chow $(X), p^{\prime}: U^{\prime} \rightarrow Z^{\prime}$ the universal family and $u^{\prime}: U^{\prime} \rightarrow X$ the cycle map. We want to prove that $U^{\prime}=Y$.

$h$ induces a morphism between the universal families $h^{\prime}: Y^{0} \rightarrow U^{\prime}$ which sits in a diagram

$$
Y^{0} \stackrel{h^{\prime}}{\rightarrow} U^{\prime} \stackrel{u^{\prime}}{\rightarrow} X \stackrel{\pi^{-1}}{\rightarrow} Y
$$

The composite is an open immersion hence birational. Thus the intermediate maps are also birational. $h^{\prime}$ is thus an isomorphism near a general fiber of $p^{0}$, and so $p^{\prime}: U^{\prime} \rightarrow Z$ is a $\mathbb{P}^{1}$-bundle over an open set. $Z^{\prime}$ parametrizes curves wich have intersection number 1 with an ample divisor. Thus $Z^{\prime}$ parametrizes irreducible curves with multiplicty 1 .

Let $E^{\prime} \subset U^{\prime}$ be the closure of $h^{\prime}\left(E^{0}\right) . E^{\prime}$ is a rational section of $p^{\prime}$ and $u^{\prime}\left(E^{\prime}\right)=$ $\{x\}$. If $E^{\prime}$ contains a whole fiber of $p^{\prime}$ then $u^{\prime}\left(E^{\prime}\right)=\{x\}$ implies that every fiber is mapped to a point which is impossible. So, $E^{\prime}$ is a section of $p^{\prime}$.

Let $B \subset U^{\prime} \backslash E^{\prime}$ be a curve such that $u(B) \in X$ is a point. Then $S:=\left(p^{\prime}\right)^{-1}\left(p^{\prime}(B)\right)$ is a ruled surface and $u^{\prime}$ contracts the curves $B$ and $E^{\prime} \cap S$ to points. By bend-andbreak (cf. [Kollár95, II.5.5.2]) this leads to a curve $C^{*} \subset X$ such that $\left(C^{*} \cdot H\right)<1$, but this is impossible. Thus $u^{\prime}$ is quasifinite on $U^{\prime} \backslash E^{\prime} . u^{\prime}$ is also birational, hence

$$
u^{\prime}: U^{\prime} \backslash E^{\prime} \rightarrow X \text { is an open embedding. }
$$

Its image contains $X \backslash\{x\}$ and it is not projective, hence $U^{\prime} \backslash E^{\prime} \cong X \backslash\{x\}$.

Let us now look at the birational map $\phi:=u^{\prime-1} \circ \pi: Y \rightarrow U^{\prime}$. Both $U^{\prime}$ and $Y$ contain $Y^{0}$ and $X \backslash\{x\}$ as open sets, and $\phi$ is the identity on $Y^{0}$ and $X \backslash\{x\}$. Thus $\phi$ is an isomorphism outside the codimension 2 sets $E \backslash E^{0}$ and $E^{\prime} \backslash E^{0}$. Since $Y / X$ has relative Picard number 1 , this implies that $\phi$ is a morphism by (9). Thus $\phi$ is an isomorphism since $\phi$ can not contract a subset of $E \cong \mathbb{P}^{n-1}$ without contracting $E$.

In particular $E^{\prime} \cong \mathbb{P}^{n-1}$. $p^{\prime}: U^{\prime} \rightarrow E^{\prime}$ is flat with reduced fibers by [Hartshorne77, Ex.III.10.9], hence it is a $\mathbb{P}^{1}$-bundle. Thus $X \cong \mathbb{P}^{n}$ by an easy argument (see, for instance, [Kollár95, V.3.7.8]).

The following lemma is essentially in [M-M64].

LEMMA 9. Let $Z_{i} \rightarrow S$ be projective morphisms with $Z_{1}$ smooth. Let $\phi: Z_{1} \rightarrow Z_{2}$ be a birational map of $S$-schemes which is an isomorphism outside the codimension 2 subsets $E_{i} \subset Z_{i}$. Assume that the relative Picard number $\rho\left(Z_{1} / S\right)$ is 1 and $Z_{1} \backslash E_{1} \rightarrow S$ is not quasifinite. Then $\phi$ is a morphism.

Proof. We may assume that $S$ is affine. Let $H_{2}$ be a relatively ample divisor on $Z_{2}$ and $H_{1}$ its birational transform. $H_{1}$ is also relatively ample because $\rho\left(Z_{1} / S\right)=1$ and $-H_{1}$ can not be relatively nef (since $H_{1}$ is effective when restricted to a positive dimensional fiber of $\left.Z_{1} \backslash E_{1} \rightarrow S\right)$. Thus $\left|m H_{2}\right|$ and $\left|m H_{1}\right|$ are both base point free for $m \gg 1$ and these are the birational transforms of each other by $\phi$. Let $\Gamma \subset Z_{1} \times_{S} Z_{2}$ be the closure of the graph of $\phi$ with projections $\pi_{i}: \Gamma \rightarrow Z_{i}$. If $\pi_{1}^{-1}(z)$ is positive dimensional then every member of $\left|m H_{2}\right|$ intersects $\pi_{2}\left(\pi_{1}^{-1}(z)\right)$, 
thus $\left|m H_{1}\right|=\phi^{-1}\left|m H_{2}\right|$ has $z$ as its base point, a contradiction. Thus $\pi_{1}$ is an isomorphism and $\phi$ is a morphism.

10 (Proof of (3)). By [Mori79] there is a rational curve $C \subset X$ such that $0<\left(-K_{X} \cdot C\right) \leq n+1$. Fix an ample divisor $L$ and pick a rational curve $C \subset X$ such that $0<\left(-K_{X} \cdot C\right)$ and $(L \cdot C)$ is minimal. Then $\left(-K_{X} \cdot C\right)=n+1$ by (3.1) and $C$ is not numerically equivalent to a reducible curve whose irreducible components are rational. Thus, as in (7), we obtain that the Picard number of $X$ is 1 and $-K_{X}$ is ample. Set $H:=-\frac{1}{n+1} K_{X} . H$ is a $\mathbb{Q}$-divisor such that $\left(H^{n}\right) \geq 1$ and $(H \cdot C) \geq 1$ for every rational curve $C \subset X$. It is easy to see that the proof of (8) works for $\mathbb{Q}$-divisors satisfying these assumptions. Thus we obtain (3).

Returning to the proof of (1), we are left with the cases when $-\left(C \cdot K_{X}\right)=n$. Then we have an at least $(n-2)$-dimensional family of curves through every $x \in X$. If there is an $x \in X$ such that all curves of degree 1 through $x$ cover $X$, then we obtain that $X \cong \mathbb{P}^{n}$ and this leads to a contradiction. Thus for every $x \in X$ the curves $\left\{C_{t}: x \in C_{t}\right\}$ sweep out a divisor $B_{x}$. The divisors form an algebraic family for $x$ in a suitable open set $X^{0} \subset X$.

11 (Case 2: $-\left(C \cdot K_{X}\right)=n$ and $B_{x_{1}} \cap B_{x_{2}} \neq \emptyset$ for $\left.x_{1}, x_{2} \in X^{0}\right)$. By assumption, any two points of $X$ are connected by a chain of length 2 of the form $C_{t_{1}} \cup C_{t_{2}}$, thus $X$ has Picard number 1 by [Kollár95, IV.3.13.3]. Thus we see that $-K_{X} \equiv n H$. The computation of the genus of a general complete intersection curve of members of $m H$ for odd $m \gg 1$ shows that $\left(H^{n}\right)$ is even. Thus $\left(H^{n}\right) \geq 2$.

Pick general $x_{1}, x_{2}$ such that there is no degree 1 curve through $x_{1}$ and $x_{2}$ and pick curves $C_{i} \supset x_{i}$ such that $C_{1} \cap C_{2} \neq \emptyset$. We can view $C_{1} \cup C_{2}$ as the image of a map $f: \mathbb{P}^{1} \vee \mathbb{P}^{1} \rightarrow X$ where $\mathbb{P}^{1} \vee \mathbb{P}^{1}$ denotes the union of 2 lines in $\mathbb{P}^{2}$. Let $y_{i} \in \mathbb{P}^{1} \vee \mathbb{P}^{1}$ be a preimage of $x_{i}$. By the usual estimates (cf. [Kollár95, II.1.7.2]) we obtain that

$$
\operatorname{dim}_{[f]} \operatorname{Hom}\left(\mathbb{P}^{1} \vee \mathbb{P}^{1}, X, y_{i} \mapsto x_{i}\right) \geq 2 n+n-2 n=n .
$$

On the other hand, these maps correspond to pairs of curves $C_{1}^{\prime} \cup C_{2}^{\prime}$ where $C_{i}^{\prime}$ passes through $x_{i}$ and $C_{1} \cap C_{2} \neq \emptyset$. By our assumption these form an $n$-2-dimensional family. The automorphism group Aut $\left(\mathbb{P}^{1} \vee \mathbb{P}^{1}, y_{1}, y_{2}\right)$ accounts for the missing 2 dimensions. Viewing $\mathbb{P}^{1} \vee \mathbb{P}^{1}$ as a reducible plane conic, [Kollár95, II.1.7.3] implies that there is an at least $(n-1)$-dimensional family of degree 2 rational curves $\left\{A_{s} \subset X: s \in S\right\}$ which pass through both of $x_{1}, x_{2}$.

As in Case 1, we obtain that

$$
\begin{array}{lll}
H^{0}\left(X, \mathcal{O}_{X}(m H) \otimes I_{x_{1}}^{m+1} \otimes I_{x_{2}}^{m+1}\right) & \geq \text { (const) } \cdot m^{n} & \text { if }\left(H^{n}\right)>2, \text { and } \\
H^{0}\left(X, \mathcal{O}_{X}(m H) \otimes I_{x_{1}}^{m} \otimes I_{x_{2}}^{m}\right) & \geq \text { (const) } \cdot m^{n-1} & \text { if }\left(H^{n}\right)=2 .
\end{array}
$$

Pick any member $D \in\left|\mathcal{O}_{X}(m H) \otimes I_{x_{1}}^{m+1} \otimes I_{x_{2}}^{m+1}\right|$ (resp. $\left.D \in\left|\mathcal{O}_{X}(m H) \otimes I_{x_{1}}^{m} \otimes I_{x_{2}}^{m}\right|\right)$ and take $A_{s} \not \subset D$. Then $\left(A_{s} \cdot D\right)=2 m$. On the other hand, $\left(A_{s} \cdot D\right) \geq \sum_{y \in A_{s}}$ mult $y \geq$ $2 m+2$ (resp. $\geq 2 m$ ). Thus $\left(H^{n}\right)=2,\left(A_{s} \cap D\right)=\left\{x_{1}, x_{2}\right\}$ and the linear system $\left|\mathcal{O}_{X}(m H) \otimes I_{x_{1}}^{m} \otimes I_{x_{2}}^{m}\right|$ is constant along $A_{s}$ for general $s$.

We also see that for general $A_{s}$, the line bundle $\mathcal{O}_{A_{s}}(m H)$ has a section with an $m$-fold zero at two general points of $A_{s}$. Since $\operatorname{deg} \mathcal{O}_{A_{s}}(m H)=2 m$, this implies that a general $A_{s}$ is a smooth rational curve.

Set $Y=B_{x_{1} x_{2}} X$ with exceptional divisors $E_{1}, E_{2}$ and projection $\pi: Y \rightarrow X$. Set $M=\pi^{*} H-E_{1}-E_{2}$. Then $|m M|=\left|\mathcal{O}_{X}(m H) \otimes I_{x_{1}}^{m} \otimes I_{x_{2}}^{m}\right|$. As in Case 1, we obtain an open set $Y^{0} \subset Y$ and a $\mathbb{P}^{1}$-bundle $Y^{0} \rightarrow Z^{0}$ with two sections $E_{i} \cap Y^{0}$. Construct 
$p^{\prime}: U^{\prime} \rightarrow Z^{\prime}$ and $u: U^{\prime} \rightarrow X$ using $\operatorname{Chow}(X)$ as before. Let $z \in Z^{\prime}$ be any point and $A_{z} \subset X$ the 1-cycle corresponding to $z$. $A_{z}$ has degree 2 and it passes through the points $x_{1}, x_{2}$. We have assumed that there is no degree 1 curve passing through $x_{1}, x_{2}$, thus either $A_{z}$ is irreducible and $\left(H \cdot A_{z}\right)=2$ or $A_{z}$ has 2 different irreducible components $A_{z}^{1} \cup A_{z}^{2}$ and $\left(H \cdot A_{z}^{i}\right)=1$.

The key point is to establish that the cycle map $u$ is quasifinite on $U^{\prime} \backslash\left(E_{1}^{\prime} \cup E_{2}^{\prime}\right)$. Assuming the contrary, we have a normal surface $S$ and morphisms $p^{\prime}: S \rightarrow Z^{*} \subset Z^{\prime}$ and $u: S \rightarrow X$ such that every fiber of $p^{\prime}: S \rightarrow Z^{*}$ is either $\mathbb{P}^{1}$ or the union of 2 copies of $\mathbb{P}^{1}$ and $u$ contracts $E_{i} \cap S$ and another curve $B$ to points.

A version of bend-and-break (12) establishes that in this case $u\left(p^{\prime-1}(z)\right)$ is independent of $z \in Z^{*}$. This is, however, impossible since $Z^{\prime} \rightarrow \operatorname{Chow}(X)$ is finite.

Again using (9) we conclude that $U^{\prime} \cong Y$ and $Z^{\prime} \cong E_{i}^{\prime} \cong \mathbb{P}^{n-1}$. Therefore

$$
\mathcal{O}_{Y}\left(\pi^{*} H-E_{1}-E_{2}\right) \cong p^{\prime *} \mathcal{O}_{E_{i}^{\prime}}(1) \cong p^{\prime *} \mathcal{O}_{\mathbb{P}^{n-1}}(1)
$$

is generated by global sections. Pushing these sections down to $X$ and varying the points $x_{i}$ we obtain that $\mathcal{O}_{X}(H)$ is generated by $n+2$ global sections. Thus $|H|$ gives a finite morphism $X \rightarrow \mathbb{P}^{n+1}$ whose image is a quadric since $\left(H^{n}\right)=2$. Thus $X$ is a smooth quadric.

LEMma 12 (3 point bend-and-break). Let $S$ be a normal and proper surface which is a conic bundle (that is, there is a morphism $p: S \rightarrow A$ such that every (scheme theoretic) fiber is isomorphic to a plane conic). Let $E_{1}, E_{2} \subset S$ be disjoint sections of $p$ and $B \subset S$ a multisection. Assume that every singular fiber $F_{a}$ of $p$ has 2 components $F_{a}=F_{a}^{1} \cup F_{a}^{2}$, and $\left(F_{a}^{i} \cdot E_{i}\right)=0$ for $i=1,2$. Let $L$ be a nef divisor on $S$ such that $\left(L \cdot F_{a}^{i}\right)=1$ for every $F_{a}$ and $i$ and $\left(L \cdot E_{1}\right)=\left(L \cdot E_{2}\right)=(L \cdot B)=0$. Then $S \cong A \times \mathbb{P}^{1}$, the $E_{i}$ are flat sections and $B$ is a union of flat sections.

Proof. The Picard group of $S$ is generated by the classes $F_{a}^{i}, E_{1}$ with rational coefficients (cf. [Kollár95, IV.3.13.3]). From the conditions $\left(L \cdot F_{a}^{i}\right)=1$ we conclude that $L=E_{1}+E_{2}+d F$ for some $d$ (and $\left.\left(E_{1}^{2}\right)=\left(E_{2}^{2}\right)\right)$. $\left(L^{2}\right) \geq 0$ and $\left(L \cdot E_{i}\right)=0$, so by the Hodge index theorem $\left(E_{i}^{2}\right) \leq 0$. Hence we conclude that $d=-\left(E_{i}^{2}\right) \geq 0$. This implies that

$$
(L \cdot B)=\left(E_{1} \cdot B\right)+\left(E_{2} \cdot B\right)+\operatorname{deg}(B / A) \cdot\left(-E_{1}^{2}\right),
$$

All terms on the right hand side are nonnegative. Thus $\left(E_{1}^{2}\right)=\left(E_{2}^{2}\right)=0$. Since $\left(E_{1} \cdot E_{2}\right)=0$, this implies that $E_{1}$ and $E_{2}$ are algebraically equaivalent. Thus there are no singular fibers.

13 (Case 3: $-\left(C \cdot K_{X}\right)=n$ and $B_{x_{1}} \cap B_{x_{2}}=\emptyset$ for general $\left.x_{1}, x_{2}\right)$. Take a general $x_{1} \in X$ and a general $x_{3} \in B_{x_{1}}$. If $\operatorname{dim}\left(B_{x_{1}} \cap B_{x_{3}}\right)=n-2$ then $\operatorname{dim}\left(B_{x_{1}} \cap B_{x_{2}}\right)=n-2$ for a general $x_{2}$, a contradiction. Thus $B_{x_{1}}$ and $B_{x_{2}}$ are either disjoint or they coincide for $\left(x_{1}, x_{2}\right)$ in an open subset of $X \times X$. The algebraic family of divisors $B_{x}$ thus determines a morphism $p: X \rightarrow A$ to a smooth curve $A$. A general fiber of $p$ is $B_{x}$. Let $Y$ be a general fiber of $p$ and $x \in Y$ a general smooth point. We have an $(n-2)$-dimensional family of curves $C_{t}$ through $x$ and all of these are contained in $Y$. $\left(C_{t} \cdot H\right)=1$ and the Picard number of $Y$ is 1 as before. Thus $-K_{Y} \cong-\left.\left.K_{X}\right|_{Y} \cong n H\right|_{Y}$. Let $\pi: \bar{Y} \rightarrow Y$ denote the normalization of $Y$. The curves $C_{t}$ lift to $\bar{Y}$, and

$$
-K_{\bar{Y}}=-K_{Y}+(\text { conductor of } \pi) \text {, }
$$


where the conductor of $\pi$ is an effective divisor which is zero iff $\pi$ is an isomorphism (this is a special case of duality for finite morphisms, cf. [Hartshorne77, Ex.III.7.2]). Thus

$$
-\left(K_{\bar{Y}} \cdot \pi^{*} H^{n-2}\right) \geq-\left(K_{Y} \cdot H^{n-2}\right)=n\left(\left.H\right|_{Y}\right)^{n-1}=n\left(\pi^{*} H\right)^{n-1} .
$$

So $\bar{Y} \cong \mathbb{P}^{n-1}$ by (8). Furthermore, we also obtain that $-K_{\bar{Y}}=n \pi^{*} H+$ (conductor), which implies that the conductor of $\pi$ is zero. Thus $Y \cong \mathbb{P}^{n-1}$ and $p: X \rightarrow A$ is generically a $\mathbb{P}^{n-1}$-bundle. This implies that $p$ has a section (cf. [Serre79, X.6-7]), hence every fiber of $p$ has a reduced irreducible component.

Let $W \subset X$ be a reduced irreducible component of a fiber of $p$ and $x \in W$ a general smooth point. The above argument applies also to $W$, and we obtain that $W \cong \mathbb{P}^{n-1}$ and $W$ is a connected component of its fiber. This shows that $X$ is a $\mathbb{P}^{n-1}$-bundle over $A$.

AcKnowledgments. Partial financial support was provided by the NSF under grant numbers DMS-9800807 and DMS-9970855.

\section{REFERENCES}

[A-B-W93] M. Andreatta, E. Ballico, Aivd J. Wiśniewski, Two theorems on elementary contractions, Math. Ann., 297 (1993), pp. 191-198

[Batyrev82] V. BATYREv, Boundedness of the degree of multidimensional toric Fano varieties, in Vestnik MGU, 1982, pp. 22-27; English translation: Moscow Univ. Math. Bull., 37 (1987), pp. 28-38.

[Fujita87] T. FUJITA, On polarized manifolds whose adjoint bundles are not semipositive, in Adv. Stud. Pure Math. 10, Algebraic Geometry, Sendai, 1985, T. Oda, ed., Kinokuniya, North-Holland, 1987, pp. 167-178.

[Hartshorne77] R. HARTshorne, Algebraic Geometry, Graduate Text in Math. 52, Springer-Verlag, Berlin, Heidelberg, New York, 1977.

[H-K57] F. HIRzEBRUCH AND K. KodAIRA, On the complex projective spaces, J. Math. Pure Appl., 36 (1957), pp. 201-216.

[Ionescu86] P. IONESCU, Generalized adjunction and applications, Math. Proc. Cambr. Phil. Soc., 99 (1986), pp. 457-472.

[Kachi99] Y. KACHI, Characterization of Veronese varieties and the Hodge index theorem, Preprint, 1999.

[Kachi-Sato99] Y. KACHI AND E. SATO, Polarized varieties whose points are joined by rational curves of small degrees, Illinois J. Math., 43 (1999), pp. 350-390.

[K-O72] S. KoBAYASHI AND T. OCHIAI, Characterization of the complex projective space and hyperquadrics, J. Math. Kyoto U., 13 (1972), pp. 31-47.

[Kollár91] J. KollÁR, Extremal rays on smooth threefolds, Ann. Sci. ENS, 24 (1991), pp. 339-361.

[Kollár95] J. KolláR, Rational Curves on Algebraic Varieties, Springer-Verlag, 1995.

[M-M64] T. MATSUSAKA AND D. MUMFORD, Two fundamental theorems on deformations of polarised varieties, Amer. J. Math., 86 (1964), pp. 668-684.

[Megyesi98] G. MEGYESI, Fano threefolds in positive characteristic, J. Alg. Geom., 7 (1998), pp. 207-218.

[Mori79] S. MoRI, Projective manifolds with ample tangent bundles, Ann. of Math., 110 (1979), pp. 593-606.

[Sato99] E. SAто, Adjoint bundles of ample vector bundles in any characteristic, J. Math. Kyoto Univ., to appear.

[Serre79] J.-P. SerRe, Local Fields, Springer Verlag, 1979.

[S-B97] N. I. Shepherd-BARRon, Fano threefolds in characteristic $p$, Comp. Math., 105 (1997), pp. 237-265.

[Siu-Yau80] Y.-T. SIU AND S. T. YAU, Compact Kähler manifolds of positive bisectional curvature, Inv. Math., 59 (1980), pp. 189-204.

[Ye-Zhang90] Y. G. YE AND Q. ZHANG, On ample vector bundles whose adjunction bundles are not numerically effective, Duke Math. J., 60 (1990), pp. 671-687. 
